\title{
EAl Endorsed Transactions

\section{Powering environment monitoring Wireless Sensor Networks: A review of design and operational challenges in Eastern Africa}

\author{
Maximus Byamukama ${ }^{1, *}$, Geofrey Bakkabulindi ${ }^{1}$, Björn Pehrson $^{2}$, Mary Nsabagwa $^{1}$ and Roseline Akol $^{1}$ \\ ${ }^{1}$ Makerere University, College of Engineering, Design, Art and Technology, P.O Box 7062, Kampala, Uganda \\ ${ }^{2}$ University of Bergen, Geophysical Institute, Postboks 7803, 5020 Bergen
}

\section{Abstract}

This paper discusses the various design and operational challenges that we have met in providing power to Wireless Sensor Networks (WSNs) deployed in environment monitoring in East Africa. While such deployments in African environments have a major advantage of abundant sunshine, both in intensity and duration, the same environments still present a number of unique challenges. With the various research initiatives in Africa being quite disjointed, a major problem is the lack of a unified knowledge base that communicates the current challenges and solutions in designing power systems for these WSNs. We implemented a WSN with several autonomous sensors and two types of gateways. We kept track of voltages, uptime and other diagnostic data. We combine our experiences from the study of these devices, and those of some others in the region and other developing countries and make recommendations where we have achieved good results and propose solutions where work is still in progress.

Keywords: Automatic Weather Station; Environment Monitoring; Power Management; Wireless Sensor Networks

Received on 07 December 2017, accepted on 19 February 2018, published on 20 March 2018

Copyright (C) 2018 M. Byamukama et al., licensed to EAI. This is an open access article distributed under the terms of the Creative Commons Attribution licence (http://creativecommons.org/licenses/by/3.0/), which permits unlimited use, distribution and reproduction in any medium so long as the original work is properly cited.

doi: 10.4108/eai.13-7-2018.155570

\section{Introduction}

The use of Wireless Sensor Networks (WSNs) in monitoring weather has become increasingly popular. They are easily scalable, eliminate the single point of failure typical in wired systems and ease the introduction of redundant nodes for back up. They are also much easier to deploy in remote and hostile environments-for example, they can be parachuted into impenetrable forests or large water bodies. A WSN consists of distributed microcontrollers with radio modules, called nodes or motes, and various sensors attached to collect data and transmit it to a central point called the sink node. The sink node is connected to an uplink device, which is used to transmit data to a remote server. There may be multiple sink nodes in a network. Figure 1 illustrates the concept of a WSN.

A set of nodes measuring various weather elements in a WSN constitutes an Automatic Weather Station (AWS). In Africa, there is an increasing amount of work dedicated to the use of WSNs in weather monitoring and agriculture through trainings, projects and inter-agency partnerships [1] [2] [3] [4] [5]. The realization that powering WSNs in developing countries presented unique challenges started as early as 2010 [6]. In light of the broad research issues and challenges involved in the design of WSNs globally, including but not limited to hardware and software platforms, operating systems, synchronization, security and cost, power-related challenges appear to be the most important [7] [8].

*Corresponding author. Email:maximus.byamukama@cedat.mak.ac.ug 

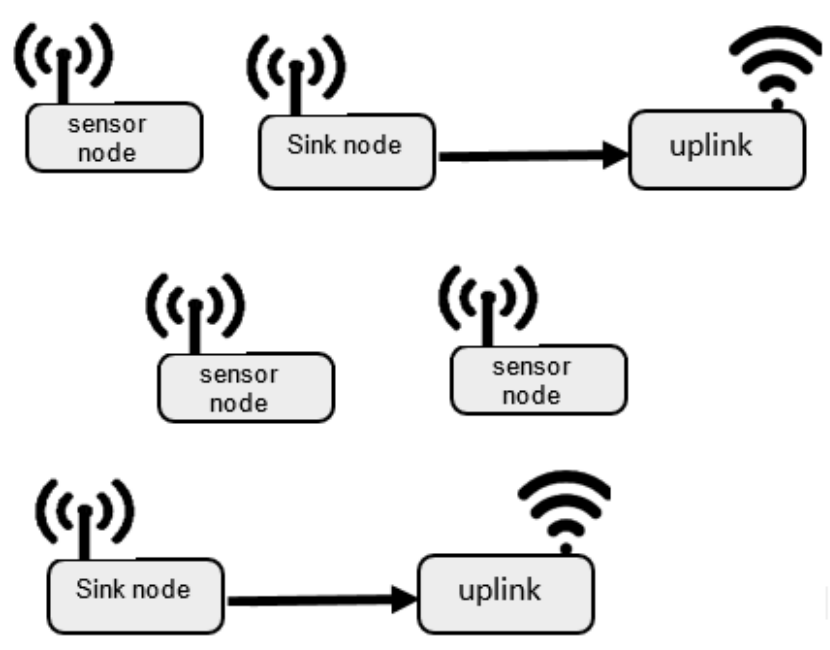

Figure 1 Illustration of a Wireless Sensor Network

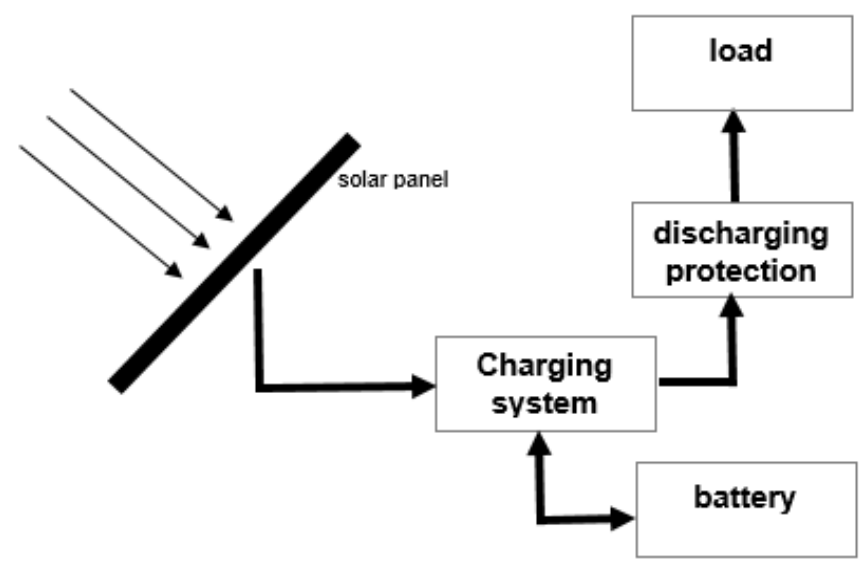

Figure 2 Illustration of a Wireless Sensor Network

Figure 2 shows the architecture of the power system of a single node in a WSN. Each node is typically autonomous, with its own energy-harvesting unit, such as a solar cell, an energy storage device, typically a rechargeable battery or supercapacitor and the corresponding power electronics to prevent over-charge, over-discharge and ensure proper operating voltages. In the East African region in particular, the lessons learned have cut across each of these components during both design and operation. We aimed to address challenges in energy harvesting, energy storage and power management. Figures 3 and 4 show actual node deployments at a weather station in Kampala.

The rest of this paper is organized as follows. Section 2 discusses the methods we have used to arrive at these findings. Section 3 discusses the design challenges faced before realizing operational WSNs. Section 4 presents the operational challenges in powering implemented sensor networks in the region, for which we recommend solutions that have yielded a satisfactory deployment. Section 5 presents other issues in powering these devices and Section 6 concludes and gives an overview of pending problems that still need to be addressed.

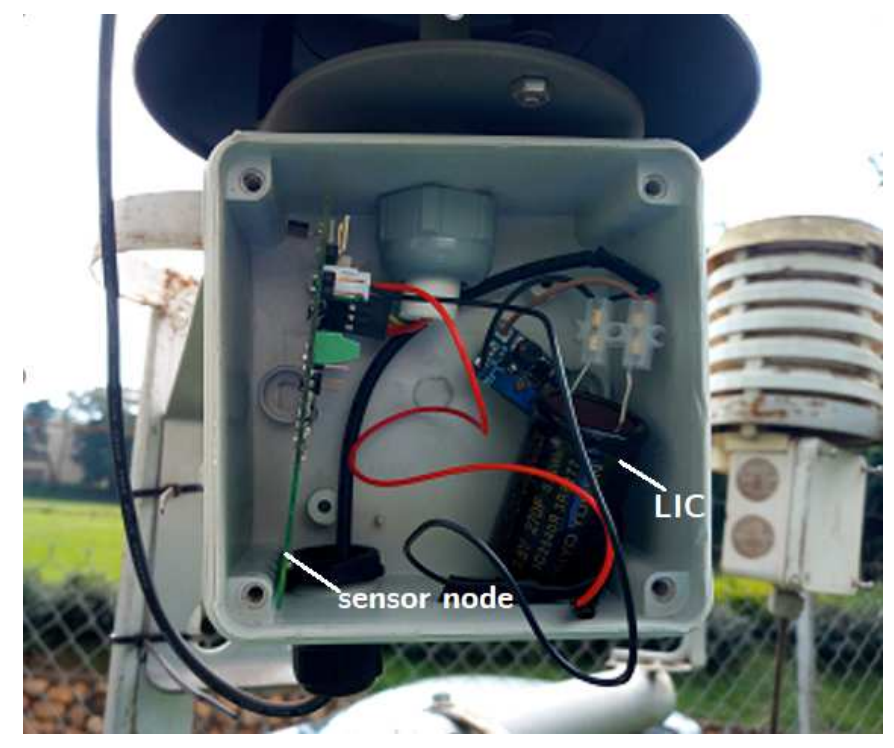

Figure 3 A wireless sensor node measuring temperature and relative humidity. It is powered by a Lithium-ion Capacitor (LIC), which is charged by a $2.5 \mathrm{~V}$ solar panel (not shown), whose voltage is passed through a boost converter to deliver $3.8 \mathrm{~V}$

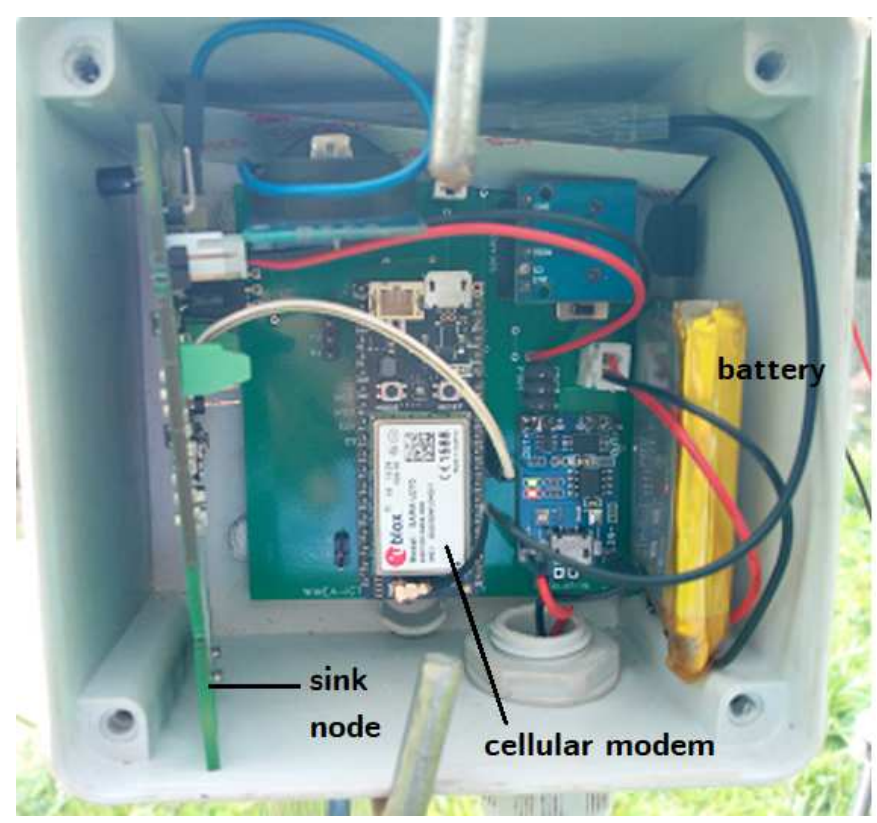

Figure 4 The gateway consisting of the sink node (left) and cellular uplink device (central) powered by a Li-ion battery (right)

\section{Methodology}

We designed and developed a WSN-based environmentmonitoring device and deployed prototypes in Bergen, Kampala, Dar-es-salaam and Juba. The deployments consist of four sensor nodes. One installed at a height of $2 \mathrm{~m}$, measuring temperature and humidity. One with sensors installed at $10 \mathrm{~m}$, measuring wind speed, direction and solar insolation. A ground node, just $0.5 \mathrm{~m}$ off the 
ground, measuring soil moisture, temperature and rainfall. The last is the sink node, which also measures atmospheric pressure. All nodes keep track of the input battery voltage, microcontroller voltage and uptime. They give notifications when power is running low. The nodes are all on-campus and are monitored by human observers from time to time to look out for any new general developments. Most diagnostic information is obtained from data sent by the gateways to a central repository over cellular or campus networks.

We have documented our experiences in over one year in this study and compared and contrasted with the work we looked at in east Africa in [1] [2] [4] [6] [9]. The review of this literature gives a broader insight given the limited geographical scope of the work. The combined approach has led to the development of a compendium of challenges faced both during the design phase and in the operational phase after deployment.

\section{Design Challenges}

\subsection{Reducing Power Consumption}

A node in a WSN that consumes more power subjects the battery to larger depth of discharge (DoD). Battery cycle life is typically stated at $80 \%$ DoD and cycle life is increased if the battery undergoes only partial discharge before being recharged. Reducing power consumption is a challenge because it often comes at the cost of reducing functionality. As such, a balance must be established between the amount of power consumed and, for example, the extent to which the system complies with frequency of reporting weather data as specified by the World Meteorological Organization.

Power consumption in WSNs must be looked at from the perspective of both the transmitter and sink nodes. We have used two main approaches to reduce power consumption. In regard to the transmitter nodes, it is often the case that they do not need to be active all the time since they report data only occasionally. Ultra-low power operation can be achieved by putting the sensor nodes to sleep and only waking them up to make measurements after some minutes. This technique has proved to be very reliable, and we have some nodes that have not run out of power for over 2 years using a single $270 \mathrm{~F}$ supercapacitor and a $4 \mathrm{~cm} \mathrm{x} 4$ $\mathrm{cm} 0.5 \mathrm{~W}$ solar PV cell. Without recharge, the sensor node (also called a mote) has been shown to run for 6 weeks [9]. With the sink node, there is often a connection to an uplink device connecting to the internet, and the whole system is called a gateway. The power consumption challenge is much greater in the sink node because it is typically always on to receive transmitted data, and the gateway has cellular, Wi-Fi or Ethernet connectivity.

Our recommendation in this case is to select low power microcontroller platforms that can be put to sleep and to select data upload intervals that reduce the power budget whenever possible. Other considerations like voltage and frequency of operation must be made. The full guidelines on low power design for environment monitoring WSNs have been published in [10]. We have been able to implement a reliable $55 \mathrm{~mW}$ system running sensor nodes based on the ATMEGA256RFR2 microcontroller from Radio Sensors AB [11] and the Electron 3G from Particle [12]. It is powered by a $2000 \mathrm{mAh}$ battery and a $2 \mathrm{~W}$ solar panel.

\subsection{Sizing Solar Panels}

We have observed that traditional solar PV panel sizing techniques underestimate the required panel size by far. We believe this is because factors like seasonal variations of solar irradiance, short-term solar irradiance variations due to cloudy days and intermittent sunshine, solar panel temperature and soiling. When designing the power supply of these WSNs, the design challenge is how to accurately size the solar panel before deployment. Solar panel sizing is of paramount importance because the consequences of a power black-out due to insufficient energy harvesting are immediate and grave. For systems that will be very remote, post-deployment visits may be impossible and the survivability of the AWS depends on its ability to retain a sufficient battery charge even in very low sunshine conditions.

In the design phase, rather than estimating the solar panel size using the traditional technique of comparing total load consumption, hours of sunshine and panel peak wattage, we recommend an actual measurement of the daily solar irradiance and the corresponding net solar panel power output by looking at the battery state of charge (SOC) and deriving how the SOC will be affected in future using historic weather data. This work is in progress, but has already shown reliable predictions of battery SOC for various solar panel wattages for a given load [13].

\subsection{Battery Selection}

When the WSN architecture has been finalized and hardware platforms decided upon, the selection of the optimal energy storage option becomes a major task. On one hand, technologies such as supercapacitors have advantages of charging very fast and having unlimited recharge cycles but cannot keep a constant voltage profile during discharge. Some types of supercapacitors exhibit a high self-discharge and loses energy even during the inactivity period of the WSN. As such, some of their stored energy is unavailable for use when their voltage goes below the operating range of the WSN microcontrollers.

While there is some recent related work on the various battery technologies available for use in WSNs, such as in [14], there has previously been a research gap in determining the criteria to follow when selecting the right battery to deploy for a given WSN use case in a given environment. In [15], we have listed down these criteria and provided a detailed discussion on selecting the optimal energy storage option for the transmitter sensor nodes, low power and high power gateways. In particular, the challenge is usually in the

i. nominal operating voltage as dictated by the microcontroller peripherals used in the node. 
ii. energy and power density, i.e. the volume of space occupied by the battery versus how much energy it can actually provide, and

iii. need for some level of regular maintenance will be required.

iv. nature of the discharge curve. Flat discharge profiles mean the battery will keep a fairly constant voltage and all energy will be available for use by the sensor node until the battery runs out.

\section{Operational Challenges}

\subsection{Charge-Discharge Regulation}

When using traditional battery technologies like Li-ion and lead acid, the protection devices for these are available off the shelf as single modules that monitor the charge and discharge cycles and stop them when the thresholds are reached. However, for technologies like Lithium Iron Phosphate $\left(\mathrm{LiFeSO}_{4}\right)$ batteries and Li-ion capacitors (LICs), the battery protection is the sole responsibility of the implementer. As such, the WSN firmware must include voltage-checking mechanisms to ensure the system remains above the minimum permitted voltage level and below the maximum to prevent destruction of the battery. The disadvantage with this technique is that buggy firmware, especially in single-threaded systems, may cause the microcontroller to hang in its active high-power state. This is often a possibility because the needs and requirements of WSNs are usually end-user specific and firmware is written iteratively for particular use cases.

Our solution to this challenge has been to use a multithreaded environment, Contiki [16], and implement battery-checking tasks to run occasionally and put the system in a very low power state when the battery goes low. This is still not sufficient, because there is still some consumption in this low power state and going below the minimum permitted voltage is still a possibility, if the battery never gets to recharge in time. The better solution is to develop custom independent hardware-based protection electronics. This, of course, needs engineering skill, costs more money and some time is diverted away from developing the actual WSN application.

\subsection{Intermittent Sunshine}

Intermittent sunshine in some seasons may cause a challenge in energy harvesting when using batteries with relatively high internal resistance. In such cases, the solar panel cannot impart all the collected energy in the short time the sunshine was available because the battery can only charge at a given rate safely.

We have solved this by using supercapacitors in some deployments. Because of the very low internal resistance, they accumulate all the available energy as and when it is available.

\subsection{High Temperatures for Batteries}

Many areas in Africa, for example South Sudan and Northern Uganda, and most of the lower Sahara region experience prolonged temperatures in the range of $25^{\circ}$ $40^{\circ} \mathrm{C}$ during the day in hot seasons. While these temperatures are acceptable for operating most battery types, operating at temperatures much higher than those in the open air must be considered because the whole power system, except the solar cell, is typically deployed inside some form of air and watertight casing as shown in Figures 3 and 4 . The lack of ventilation can cause the temperatures inside to rise several degrees higher. Figure 5 shows an actual plot showing the variation of air temperature inside a typical white PVC deployment box, the soil temperature and the free air temperature outside the box.

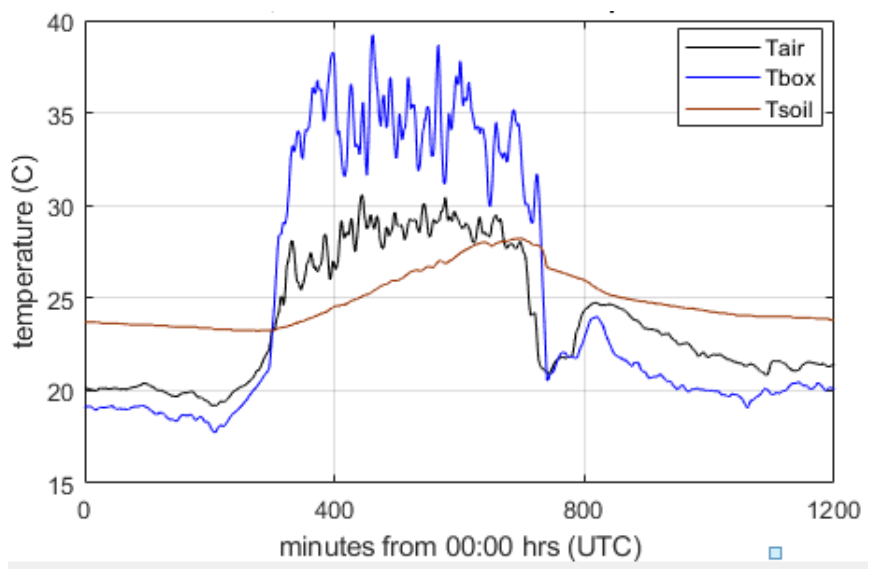

Figure 5 Variation of air temperature in free air, airtight box and soil

The temperature enables us to make some recommendations on how to handle batteries in WSNs. For high-power deployments that may need Lead-acid batteries, the battery is best deployed underground where temperatures are lower and not very dynamic. Batteries inside airtight boxes must be tolerant to large fluctuations in temperature and to temperatures $15^{\circ} \mathrm{C}$ higher than freeair temperature during the day.

The solar panels and power electronics have also shown vulnerability to some weather elements. In the work leading to [17], it was observed that the low power electronics components were destroyed by RFI energy from lightning strikes. The solution was to use transientvoltage-suppression (TVS) protection diodes at the end of the wires from the solar panels just before the connection to the power electronics. Solar panels are also of various grades and some very low cost panels showed very poor tolerance of harsh weather and gave no power output after 12 months.

\subsection{Dust Accumulation}

While we are aware that there is a reduction in power output when WSNs are deployed in a dusty environment, we have not yet quantified the impact of dust accumulation on the net power output of solar panels in various locations in Eastern Africa. In [18], this reduction is shown to be 
$32 \%$ after 8 months for Riyadh and $17 \%$ due to sand accumulation on panels after only 6 days. The huge variance suggests a need to perform a custom evaluation for a given deployment site and include this factor in the energy budget.

Our solution so far is to use human intervention to clean. A number of these AWS are to be installed close to sites of the national meteorological authorities or prominent places like schools and police stations. A small maintenance budget can be included to pay the labor hired to clean the panels regularly. To avoid this, some emerging techniques in this area are the use of self-cleaning solar panels. A review of this trend is available in [19]. Considerations, of course, must be made for cost and complexity that these mechanisms add to the WSN design.

\subsection{Quiescent Loads}

When making energy budget calculations for sizing solar cells and batteries in WSNs, it is easy to neglect quiescent power consumed by the power electronics. For example, a typical buck converter based on the LM2596 consumes up to $8 \mathrm{~mA}$. The common AMS1117 buck regulator used to step-down to $3.3 \mathrm{~V}$ in most embedded systems consumes $5 \mathrm{~mA}$ under no load. In our implementation in [10], an active node consumes $12 \mathrm{~mA}$. Such an inefficient regulator would reduce battery life by $30-40 \%$.

Our recommendation is to either use regulators with ultralow quiescent current, such as the LP2950 we are using in our sensor nodes, or to do away with regulators completely and use a battery technology whose nominal voltage is within the operating range for the sensor node and whose voltage remains fairly constant as it discharges. The Li-ion technologies meet these requirements quite well.

\section{Other Issues}

We now discuss some other issues that may come in during the design stage or during operation that we believe need consideration because of socio-economic and human factors.

\subsection{Securing Power Components}

For gateway platforms that provide versatile functionally, such as the Raspberry Pi to which one can log on remotely using SSH, or other embedded Linux computers, the size of the battery and solar panel will be considerably large. We have used a 45Ah battery and 30W solar panel for this use case. Such sizes have domestic value and the meteorological authorities in Uganda and Tanzania have reported vandalism and theft of similar solar panels and batteries to be major challenges [20] [21]. Figure 6 shows a typical way this challenge is wrongly addressed in Uganda - using a secure cage. A primary disadvantage of this technique is that the cage rods cast shadows on the surface of the panel and reduce power output.

We believe that our solution of reducing power consumption of the gateway, and thus using much smaller solar panels and batteries will fail to attract such vandalism. However, there is a general recommendation from the meteorological authorities to install, if possible, the WSNbased automatic weather stations next to secure sites, such as police stations and schools.

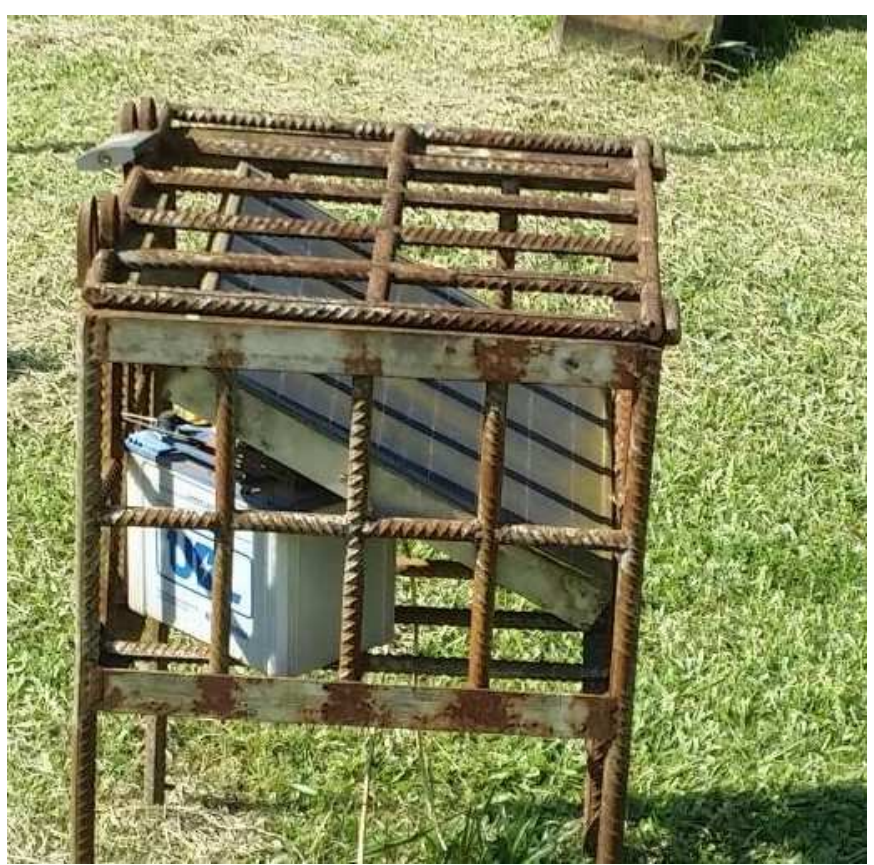

Figure 6 Securing solar panels and batteries using cages is common in East Africa

\subsection{Cost of Power}

The penetration of solar energy programmes in Africa has made the price of panels more widely affordable. There is still an issue of cost when it comes to batteries. In [15], we have mentioned the use of LICs as a good emerging battery technology to use for very low power nodes in WSNs. These, however, cost $\$ 90 / \mathrm{Wh}$. In comparison, lead acid batteries in Uganda go for about \$0.08/Wh and lithium-ion batteries for about $\$ 1 / \mathrm{Wh}$. Some research budgets may not meet the cost of using emerging energy storage technologies that could greatly benefit WSNs.

\section{Conclusions and Further Work}

We have presented the various challenges that affect the design and operation of power systems in WSNs. There is still some work to be done. In reducing power consumption, radio duty cycling protocols that could reduce the power consumed by the sink node by a large margin are typically only developed for a few platforms. There is still a lack of directed software development effort to port these protocols to other common microcontroller platforms. The lower the power consumed, the less the challenge on battery selection and solar panel sizing.

Researchers in WSNs that are going to be engineered and actually deployed on a large scale in dusty areas need to consider the new concept of Photovoltaic Soiling Index PVSI [22] and approximate the PVSI for their regions or sites. 
Finally, battery health monitoring algorithms and hardware for emerging batteries technologies, like LICs and $\mathrm{LiFeSO}_{4}$, are currently unavailable and are avenues for further research. The authors are currently working with a number of partners on some of these topics.

\section{Acknowledgements.}

We thank the Norwegian Agency for Development Cooperation for funding the WIMEA-ICT Project in East Africa under which this research was done.

\section{References}

[1] M. Mafuta, M. Zennaro, A. Bagula, G. Ault, H Gombachika and T. Chadza, "Successful deployment of a Wireless Sensor Network for precision agriculture in Malawi," in 2012 IEEE 3rd International Conference on Networked Embedded Systems for Every Application (NESEA), Liverpool, 2012.

[2] M. MASINDE, A. BAGULA and M. NZIOKA, "SenseWeather: Sensor-Based Weather Monitoring System for Kenya," in IST-Africa 2013 Conference, Nairobi, 2013.

[3] WIMEA ICT Project, Makerere University, 2013. [Online]. Available: www.wimea.mak.ac.ug.

[4] M. Zennaro, A. Bagula and M. Nkoloma, "From Training to Projects: Wireless Sensor Networks in Africa," in 2012 IEEE Global Humanitarian Technology Conference, Seattle, 2012.

[5] M. Ali, "The Use of Wireless Sensor Networks in African Agriculture," Oulu University of Applied Sciences, Oulu, 2015.

[6] M. Zennaro, H. Ntareme and A. Bagula, "On the design of a flexible gateway for wireless sensor networks," in $1 s t$ Workshop on Wireless Broadband Access for Communities and Rural Developing Regions, Karlstad, 2008.

[7] S. Sharma, R. K. Bansal and S. Bansal, "Issues and Challenges in Wireless Sensor Networks," in 2013 International Conference on Machine Intelligence and Research Advancement, Katra, 2013.

[8] A. Sharma and G. S. Tewolde, "Considerations in low power wireless sensor networks," in 2015 IEEE International Conference on Electro/Information Technology (EIT), Dekalb, 2015.

[9] R. Olsson and B. Pehrson, "Powering Devices Using Ultracapacitor batteries," in AFRICON2015, Addis Ababa, 2015.

[10] M. Byamukama, J. N. Nannono, K. Ruhinda, B. Pehrson, M. Nsabagwa, R. Akol, R. Olsson, G. Bakkabulindi and E. Kondela, "Design Guidelines for Ultra-low power Gateways in Environment Monitoring Wireless Sensor Networks," in AFRICON 2017, Cape Town, 2017.

[11] Radio Sensors AB, [Online]. Available: www.radiosensors.com. [Accessed 1 June 2018].

[12] Particle, "Electron (2G/3G/LTE) Cellular Hardware," [Online]. Available: https://www.particle.io/products/hardware/electroncellular-2g-3g-lte/. [Accessed 12 June 2018].
[13] M. Byamukama, "Solar Panel Sizing Models for WSNs in Environment Monitoring," Zenodo, June 2018. [Online]. Available: https://doi.org/10.5281/zenodo.1307150.

[14] A. Othman, "Energy storage system options in Intelligent Wireless Sensor Network," in 2017 International Conference on Military Technologies (ICMT), Brno, 2017.

[15] M. Byamukama, R. Akol, G. Bakkabulindi, B. Pehrson, R. Olsson and J. Sansa-Otim, "Energy storage options for environment monitoring Wireless Sensor Networks in rural Africa," in 2018 IEEE 12th International Conference on Compatibility, Power Electronics and Power Engineering (CPE-POWERENG 2018), Doha, 2018.

[16] "Contiki: The Open Source OS for the Internet of Things," [Online]. Available: http://www.contiki-os.org/. [Accessed 21 June 2018].

[17] A. Nungu, B. Pehrson and G. Nsubis, "Serengeti Broadband," in Proceedings of the Second ACM SIGCOMM Workshop on Networked Systems for Developing Regions, Seattle, 2008.

[18] M. Mani and R. Pillai, "Impact of dust on solar photovoltaic (PV) performance: Research status,challenges and recommendations," Renewable and Sustainable Energy Reviews, vol. 14, pp. 3124-3131, 2010.

[19] A. Syafiq, A. Pandey, N. Adzman and N. AbdRahim, "Advances in approaches and methods for self-cleaning of solar photovoltaic panels," Solar Energy, vol. 162, pp. 597$619,2018$.

[20] M. Nsabagwa, M. Byamukama, J. Sansa-Otim and R. Okou, "Network Densification Strategies for Automatic Weather Stations: Challenges and Opportunities for Uganda," in 2016 IST-Africa Week Conference, Durban, 2016.

[21] E. Kondela, A. Nungu and B. Pehrson, "Status of Existing Weather Observation Station Network in Tanzania and the Possibility to Automate and Densify it," in AFRICON, Addis Ababa, 2015.

[22] K. Menoufi, "Dust Accumulation on the Surface of Photovoltaic Panels: Introducing the Photovoltaic Soiling Index (PVSI)," Sustainability, vol. 9, no. 6, 2017. 